\title{
L'emprise de l'homophobie internalisée : soumission et autodestruction identitaire comme mécanismes de contrôle social dans Baisers cachés
}

\section{Hasheem Hakeem, Simon Fraser University}

Baisers cachés est un téléfilm réalisé par Didier Bivel qui s'articule autour de l'homophobie dans un lycée français. Formé à l'université de Paris 8, Bivel a réalisé plusieurs téléfilms, dont les plus récents, Parole contre parole (2016) et Un homme parfait (2019), traitent respectivement de questions de viol et d'inceste tout en s'interrogeant sur les rapports de pouvoir, sur la libération de parole des femmes et sur le privilège hétéromasculin. Dans plusieurs de ses films, Bivel se consacre aux questions de justice sociale et il semble offrir une tribune à ceux et celles dont la voix est souvent réduite au silence. Après sa première projection en février 2016, Baisers cachés a remporté le prix de la critique au Festival de Luchon et son réalisateur a fait le tour des télévisions belges et suisses. Le film a finalement été diffusé en prime time le 17 mai 2017 sur la chaîne publique de France 2, à l'occasion de la Journée internationale contre l'homophobie et la transphobie et a captivé environ 3,6 millions de téléspectateurs (Dubois ; Hedrich). Suivi d'un débat en direct, Homophobie, le combat continue, avec de nombreux témoins (entre autres, des jeunes gays, des parents et des responsables d'associations), le film a connu une visibilité inédite dans un contexte social où les actes homophobes sont en forte augmentation depuis l'adoption de la loi sur le mariage pour tous en France en $2013^{1}$. Par conséquent, Baisers cachés constitue en quelque sorte une réponse au climat homophobe qui règne actuellement en France, notamment dans les sphères familiale, sociale et scolaire.

Le film raconte l'histoire de Nathan (joué par Bérenger Anceaux), un jeune adolescent gay de seize ans nouvellement arrivé dans un lycée, et celle de Louis (interprété par Jules Houplain), un garçon de sa classe qui l'embrasse lors d'une soirée avant la rentrée scolaire. Le baiser est photographié par l'amoureuse de Louis sans que les deux garçons s'en aperçoivent ; toutefois, seul le visage de Nathan est visible à l'image. La photo est ensuite diffusée sur les réseaux sociaux et provoque un scandale dans le lycée où un professeur d'anglais et une enseignante de mathématiques tentent de lutter contre 1'homophobie. Sachant que Nathan est homosexuel, un groupe de garçons dispersés l'agresse dans le vestiaire, dont Louis qui, à l'instar des autres garçons visés par la rumeur entourant la photo, tente de prouver qu'il n'est pas attiré par Nathan. Après 
son « coming out », la relation entre Nathan et son père Stéphane (Patrick Timsit) est tumultueuse. Cela dit, Stéphane finit par accepter l'homosexualité de son fils, surtout lorsqu'il le retrouve dans sa chambre le visage ensanglanté. Quant à Louis, il est clairement mal dans sa peau et tente de masquer à sa famille, à ses amis et à sa copine Laura son homosexualité et son désir amoureux pour Nathan. L’intérêt de ce film, précisons-le, réside dans la pertinence de sa thématique et dans le choix du destinataire visé : les adolescent.e.s du secondaire.

Par une analyse de l'évolution du personnage de Louis, je m'intéresse particulièrement dans cet article à la manière dont les discours hétéronormatifs forment la psyché de l'individu, allant jusqu'à créer les conditions de l'homophobie internalisée et à pousser ultimement le sujet queer à l'autodestruction. L'analyse portera ainsi sur la façon dont la mise en place de l'hétéronormativité se révèle au service de la discipline et de la régulation affective du personnage de Louis qui est pris au piège dans un système qui lui donne l'illusion que sa survie dépend de sa capacité à pouvoir intégrer et performer les normes hétérosexuelles.

\section{L'école et la mise en place d'une institution hétéronormative}

Dans Baisers cachés, l'école est représentée comme une institution hétéronormative qui a pour but de produire des sujets hétéronormés. Comme le montre le film, seule l'hétérosexualité a le droit d'être hypervisible dans l'espace public, alors que la diversité sexuelle est normalement associée au secret, au tabou et à l'interdit. Ainsi, l'hétérosexualité en tant que système de pouvoir normalisateur, institutionnalise l'homophobie en dévalorisant, dans les espaces discursifs et matériels de l'école, toute identité qui ne performe pas l'hétéronormativité. Par exemple, après la circulation de la photo de Nathan et de Louis sur les réseaux sociaux, les élèves de l'école s'efforcent de deviner l'identité de l'autre garçon puisque seul le visage de Nathan est visible sur la photo. Dans ce contexte d'incertitude, plusieurs garçons sont visés par des messages écrits au tableau dans les salles de classe et chacun d'entre eux tente un par un de se défendre pour plaider son innocence. Au moment de la première accusation dans le cours d'anglais (10:29), Nathan se fait frapper sur le nez par Lucas, le premier garçon visé par cet interrogatoire pervers mené par les élèves. Lors de cette altercation, le professeur d'anglais remarque le champ lexical du crime dans le langage utilisé par les étudiant.e.s : «Vous vous rendez compte des mots que vous utilisez ? Accuser, avouer. On dirait que vous parlez d'un crime » (11:10-11:18). La conversation se poursuit lorsqu'un autre étudiant ajoute : «Il a choisi ce qu'il est ; il va l'assumer jusqu'au bout » (11:31- 
11:34). Bien que l'homosexualité ne soit plus un crime ${ }^{2}$, le discours de ces jeunes peut être lu comme un « réaménagement du pouvoir de punir » (Foucault 196) dans la mesure où le pouvoir de punir l'homosexualité dans ce milieu scolaire est profondément ancré dans le corps social (98) : « [On] ne punit donc pas pour effacer un crime, mais pour transformer le coupable » (150). La tentative de dévoiler publiquement l'identité de l'autre garçon, en écrivant plusieurs noms au tableau ou en traitant certains garçons de «pédé », serait une manière de discipliner le garçon inconnu sur cette photo, en l'occurrence Louis, et tout autre garçon queer en les forçant à rester dans le placard et en les confinant dans l'espace public à partir d'une identité hétérosexuelle. Les propos des étudiant.e.s montrent que l'homosexualité ne préexiste pas au discours social qui la définit plutôt comme une perversion, une pathologie et un crime.

La même logique s'applique dans le cas de certain.e.s enseignant.e.s et du directeur d'école qui privilégient et valorisent l'hétérosexualité. Lors d'une scène dans la salle des professeur.e.s, une enseignante raconte qu'une autre bagarre a éclaté dans sa classe autour d'un autre nom au tableau, celui de Marco, et que Nathan a été agressé. Un autre enseignant répond : «Ça m’étonnerait que ce soit lui », insinuant que Marco réussit probablement à bien performer la masculinité normative et, ce faisant, à donner l'illusion d'être hétérosexuel (17:05-17:32). Exprimant sa stupeur devant ce commentaire, Tristan, l'enseignant d'anglais, déplore le fait de ne pas pouvoir aider Nathan, alors que la même enseignante lui répond : «Tout ça, c'est de sa faute aussi. Il n'avait qu'à rester discret » (17:32-17:37). Lorsque la population générale est présumée hétérosexuelle jusqu'à preuve du contraire (l'exemple de Marco), chaque individu doit vivre d'une manière qui ne reflète pas son identité sexuelle ressentie, son affectivité, son expression de genre et ses désirs. C'est cette présupposition de l'hétérosexualité qui limite l'expression de soi et qui empêche Louis de sortir du placard parce qu'il a déjà été configuré affectivement par le discours hétérosexiste scolaire à être invisible dans l'espace public. De surcroît, précisons que ce n'est pas Nathan qui n'a pas été discret, mais Laura, la copine de Louis, qui a fait circuler la photo de ce baiser « caché » qui a eu lieu dans un jardin isolé loin du regard des autres. Ainsi, nous pouvons considérer que tout espace, qu'il soit public ou intime, est à la base soumis à la surveillance hétéronormative dans le but que le plus grand nombre possible d'êtres humains s'identifient comme étant hétérosexuels ${ }^{3}$. L'homophobie s'inscrit donc dans une logique de contrôle social identitaire qui sert à conditionner un individu à se confiner dans le placard afin d'éviter le châtiment physique, mais surtout psychologique et affectif de la société hétéronormative. Cette 
discipline est d'ailleurs renforcée par le directeur du lycée qui interdit à Tristan de parler d'homosexualité et de lutter contre l'homophobie dans ses cours, craignant de froisser certains parents (16:20-17:04). Par conséquent, dans Baisers cachés, tout l'écosystème scolaire et ses acteur.rice.s renforcent l'homophobie et rappellent que la sexualité est fondamentalement politique (Rubin 267). Dans son ouvrage récemment publié, Hétéro, l'école ? Plaidoyer pour une éducation antioppressive à la sexualité (2019), Gabrielle Richard confirme que l'école est un système de pouvoir qui privilégie les vies hétéronormées, tout en dévalorisant celles qui ne se conforment pas à l'hétéronormativité : «Et l'école, où évoluent et se côtoient l'ensemble des jeunes, est l'un des principaux lieux de transmission de ces pressions normatives, qui s'exerce autant par les pairs, par le personnel scolaire, par la culture institutionnelle que par les modèles mêmes d'éducation à la sexualité » (13).

\section{Du discours hétéronormatif à l'homophobie internalisée}

Partons du principe que la sexualité est une production sociale émanant d'un discours spécifique à une époque donnée. Dans Baisers cachés, ce sont les discours scolaires et familiaux qui produisent la configuration sociale et culturelle de l'homosexualité. Ces discours sont au service de l'homophobie internalisée, comme l'illustre l'interaction discrète qui a lieu entre Nathan et Louis après la soirée où ils se sont embrassés pour la première fois. Après avoir refusé de rappeler Nathan, Louis accepte finalement de le rejoindre dans un lieu isolé tout près d'une usine. Bien que Louis ait pris l'initiative d'embrasser Nathan lors de leur première rencontre, Louis n'exprime plus le même sentiment lorsqu'il le revoit. Nathan confie à Louis qu'il avait besoin de le voir et qu'il se sentait seul, mais au moment où il s'approche de lui pour tenter d'effleurer de sa main son visage, Louis le repousse avec vigueur et lui dit : « je suis pas comme toi, moi. Ce baiser était juste pour essayer » (26:35-26:52). Si, comme le rappelle Michel Foucault dans Surveiller et punir (1975), « [un] corps bien discipliné forme le contexte opératoire du moindre geste » (178), nous pouvons alors interpréter le geste de repousser la main de Nathan comme un acte de langage, voire une performance du discours hétéronormatif qui sert à affirmer l'hétérosexualité de Louis et à nier son homosexualité. Il s'agit également d'une performance de la masculinité parce qu'en refusant le geste de tendresse de Nathan, donc d'un autre garçon, et en affirmant qu'il ne l'a embrassé ni par désir, ni par amour, ni par affection, mais par curiosité, Louis agit en fonction de la manière dont il a été discipliné. Autrement dit, il est tiraillé entre le désir sexuel qu'il ressent 
pour Nathan et le script hétéronormatif qu'il est obligé de performer dans l'espace public en tant qu'homme hétérosexuel. Ce geste de refus à la fois discursif et performatif dans cette scène permet de constater que la discipline individuelle dans ce film est de l'ordre de l'affect. Si nous nous référons à l'approche foucaldienne, le corps de Louis est bien discipliné dans cette scène parce qu'il contrôle son affectivité queer jusqu'au point de s'interdire de ressentir ses plus intenses désirs : il refuse catégoriquement de toucher tendrement Nathan et de le désirer sexuellement et, ce faisant, il canalise cette affectivité afin de produire la performance hétérosexuelle qui est produite par la discipline sociale hétéronormative.

De plus, cette discipline du corps est également liée à la manière dont Louis s'oppose à l'homosexualité : «Je suis pas comme toi, moi ». Bien que Louis n'affirme ni son homosexualité ni son hétérosexualité, il laisse entendre qu'il n'est pas gay parce que dans un système binaire, ne pas être gay, c'est être hétérosexuel. Dans son ouvrage Epistemology of the Closet (1990), Eve Sedgwick constate que la structure binaire entre l'homosexualité et l'hétérosexualité ne produit pas seulement une opposition, mais elle met aussi en place une hiérarchie dans laquelle l'identité est définie par la négation de son altérité :

[...] categories presented in a culture as symmetrical binary oppositions heterosexual/homosexual, in this case - actually subsist in a more unsettled and dynamic tacit relation according to which, first, term B is not symmetrical with but subordinated to term A ; but, second, the ontologically valorized term A actually depends for its meaning on the simultaneous submission and exclusion of term B ; hence, third, the question of priority between the supposed central and the supposed marginal category of each dyad is irresolvably unstable, an instability caused by the fact that term B is constituted as at once internal and external to term A. (9-10)

Par conséquent, si l'hétérosexualité n'est pas définie de manière précise en elle-même, mais plutôt par la dévalorisation, l'exclusion et la marginalisation de l'homosexualité, nous pouvons alors considérer que Louis intériorise cette logique binaire afin de pouvoir échapper aux conséquences sociales qui découlent de la transgression du système hétéronormatif. Si nous suivons Sedgwick, l'hétérosexualité serait une invention de contrôle social qui puise son sens et qui existe en niant, en rejetant et en excluant l'altérité et la diversité sexuelle. De surcroît, dans Gender Trouble (1990), Judith Butler rappelle que « gender is always a doing, though not a doing by a subject who might be said to preexist the deed $\gg(34)$ et, alors, c'est la performance de la norme hétérosexuelle masculine - entre autres représentée par le refus de Louis de se laisser toucher de façon affectueuse par un autre garçon et par son intériorisation du système binaire du genre - qui produit socialement 
l'apparence de son identité hétérosexuelle. Pour ces raisons, l'hétérosexualité de Louis, telle que nous l'observons dans cette scène, n'existe que dans la temporalité de la performance ; elle est une construction sociale découlant d'une série de performances répétées des normes hétérosexuelles.

Cette performance de l'hétéronormativité est le résultat des effets de ses relations affectives, notamment lorsque l'on considère son rapport avec son père. Lors d'un souper en famille, nous constatons le malaise de Louis lorsque sa mère lui dit qu'elle a croisé la mère de Laura, la copine de Louis, et qu'elles se sont parlé de « leurs amoureux ». Étant visiblement mal à l'aise par ce commentaire, Louis tente de changer de sujet et lui dit qu'il n'épousera pas Laura (30:50-31:20). L'institution de la famille, conception encore traditionnelle et enracinée dans les fondements religieux catholiques de la société française depuis des siècles ${ }^{4}$, soumet Louis à la norme hétérosexuelle et il se retrouve emprisonné par une identité sexuelle qui ne lui permet pas d'aimer et de vivre de manière authentique. À cela s'ajoute tout le contexte social et institutionnel qui pousse Louis à nier son homosexualité, car s'il l'admet, il devient aussi victime du regard des autres, des insultes ou d'éventuelles agressions physiques. Louis, par sa réticence à dire explicitement qu'il n'est pas amoureux de Laura, demeure toujours sous l'emprise affective de ses parents et de la société française en général dans la mesure où il prend conscience du fait qu'afin de se sentir épanoui en tant qu'homme dans un monde hétéronormatif, il faut surtout s'identifier comme étant hétérosexuel, être amoureux d'une fille et performer la masculinité. Nier est alors une façon de se protéger de la menace réelle et imminente de l'homophobie. Il s'agit d'une interaction éphémère, mais extrêmement marquante parce qu'elle contribue à former des affects plus complexes, notamment la peur qui serait liée, quant à Louis, à la prise de conscience de sa subjectivité dissidente dans un contexte où la violence contre les personnes LGBTQ est encore fortement ancrée dans la fabrique sociale, malgré les avancées législatives (SOS homophobie 7). Lorsque la mère évoque le nom de Nathan et son homosexualité, Louis fait semblant de ne pas bien le connaître et évite de regarder ses parents dans les yeux. Le père ajoute que cela ne doit pas être facile pour les parents, mais rappelle toutefois à son fils de «garder ses distances », comme s'il était dans un match de boxe (31:27-31:56), discipline sportive que le père s'évertue d'ailleurs à imposer à son fils. Si nous poussons l'analyse, l'expression « garder ses distances » évoque la peur d'attraper une maladie. Rappelons que jusqu'en 1981, 1'homosexualité était considérée comme une maladie mentale en France (Agence France-Presse) et même physique pendant les années Sida, certain.e.s croyant à tort que la transmission passait par le toucher et non par le sang ${ }^{5}$. 
Pour ces raisons, l'homophobie extérieure que Louis ressent à travers ses interactions affectives, notamment avec son père qui l'entraîne pour être un boxeur, n'est pas anodine : elle donne forme à sa psyché et à sa subjectivité. L'homophobie structure physiquement Louis afin de faire de son corps discipliné un système opératoire hétéronormatif du moindre geste (Foucault 178). Le malaise dans le regard de Louis au cours de cette scène déconcerte parce que ce sentiment négatif montre à quel point la société hétéronormative est une prison psychique donnant l'illusion d'une vie heureuse et normale qui perdure sous le couvert de fausses apparences.

\section{Performance spectaculaire de l'homophobie internalisée}

Dans Baisers cachés, la valorisation de l'hétérosexualité et la dévalorisation de l'homosexualité dans le milieu familial sont ainsi intériorisées par Louis afin qu'il suive la norme homophobe. En s'appuyant sur Foucault, Brian Massumi, dans Politics of Affect (2015), théorise ce processus d'intériorisation du pouvoir dans une optique affective :

The greatest power of the law is the power to form us. Power doesn't just force us down certain paths, it puts the paths in us, so by the time we learn to follow its constraints we're following ourselves. The effects of power on us are our identity. That's what Michel Foucault taught us. If power just came at us from outside, it was just an extrinsic relation, it would be simple. You'd just run away... Power comes up into us from the field of potential. It « in-forms us », it's intrinsic to our formations, it's part of our emergence as identified individuals, and it emerges with us - we actualize it, as it in-forms us. So in a way it's as potentializing as what we call freedom, only what it potentializes is limited to a number of predictable paths. It's the calculable part of affect, the most probable next steps and eventual outcomes. As Foucault says, power is productive, and it produces not so much repressions as regularities. (18-19).

Ainsi, l'identité est le résultat des effets affectifs des relations de pouvoir, vu que chaque individu a été formé et structuré par ce pouvoir relationnel qui n'est pas répressif, mais qui produit ce que Massumi appelle des « regularities of capture » $(102)^{6}$. C'est notamment la raison pour laquelle Louis se sent emprisonné et incapable de communiquer son désir amoureux et sexuel pour Nathan. Même en l'absence de ses parents, il ne peut plus se séparer de la discipline hétéronormative, car profondément inscrite en lui. Et cette discipline l'a formé psychiquement à tel point qu'il devient, de son plein gré, l'agent principal de ce scénario hétéronormatif.

Pour constater le fonctionnement et l'ampleur de cette emprise affective, considérons le moment dans le film où Nathan, après un cours d'éducation physique, se retrouve entouré par ses agresseurs dans le vestiaire des garçons. L'un des garçons l'insulte en lui disant : « T'as pas honte ? 
Le vestiaire des meufs [femmes] est là-bas » (32:56-32:59). Alors que Nathan se lève pour quitter le vestiaire, plusieurs garçons foncent sur lui et commencent à lui donner des coups de pied. La caméra se focalise sur Louis, qui observe et contemple cette violence d'un regard presque vide d'émotions, avant de mettre à l'écart les autres garçons pour ainsi devenir le bourreau principal. Mû par les attentes d'une société hétéronormative, Louis bat Nathan de manière si intense qu'un autre garçon doit intervenir avant que celui-ci ne puisse quitter le vestiaire les larmes aux yeux et le visage ensanglanté (32:47-33:50). La performance de cette masculinité spectaculaire est dirigée vers les autres garçons, dans la mesure où c'est en performant en public cette discipline hétéronormative devant le regard masculin hétérosexuel que Louis est forcé d'affirmer socialement sa masculinité hétérosexuelle. L'homophobie extérieure, fonctionnant d'emblée comme un « panoptisme ${ }^{7}$ affectif, est ensuite internalisée et finalement actualisée au moment où Louis bat Nathan, au prix de mensonges et du saccage de son affectivité amoureuse et sexuelle.

Si nous suivons la logique de Massumi, il existe un nombre limité de possibilités qui peuvent être actualisées par Louis au moment où il est confronté de manière frontale à l'agression du garçon qu'il aime. Mais en choisissant de battre Nathan plutôt que de venir à son secours, Louis actualise une réalité par laquelle il est interpellé affectivement, mais qui s'avère pourtant prévisible et en quelque sorte prédéterminée dans un monde hétéronormatif où, pour survivre, il doit détruire le garçon qu'il aime afin de ne pas aller vers l'autre possibilité, qui serait d'assumer pleinement son amour et son désir pour Nathan. En fait, la marginalisation, la stigmatisation et l'exclusion de Nathan pourraient être interprétées comme un avertissement qui serait dirigé à tous ceux qui, comme Louis, seraient tentés de se livrer à leurs désirs homosexuels et d'oser les affirmer publiquement. L'homophobie est certes un potentiel - pour reprendre l'expression de Massumi qui doit être activé volontairement par Louis, mais cela ne veut pas dire qu'il devient conscient de sa complicité dans ce processus d'assujettissement insidieux. Massumi écrit :

The dominated classes must be induced to mistake their own interests for the mirage of the « general » interest - and do so with passion. They must be duped into affectively investing in the mechanisms of power that oppress them, without ever noticing the contradiction. They must become the willing instruments of their own domination. This is most efficiently done by weaving ways of feeling and acting that are in consonance with the power structure of society into the habitual fabric of everyday life, where they go on working, unexamined. Ideology works best when its structure of ideas is lived-acted out in the everyday, without being thought out. $(85)$ 
Pour que l'homophobie puisse véritablement influer sur sa manière d'entendre, d'agir et d'être au monde, Louis doit devenir un dispositif du système disciplinaire et régulateur hétéronormatif qui l'opprime en actualisant ce qui lui a donné forme psychiquement et affectivement. En d'autres termes, « [power] hooks into the individual through feelings, and then pulls the strings that lead the individual into deluded acquiescence to its assigned role » (Massumi 86). Ayant intériorisé dans son milieu familial, social et scolaire des «ways of feeling and acting » (85) conformes aux normes de la société hétéronormative, Louis est pris affectivement dans une chorégraphie de l'hétérosexualité dont il devient lui-même l'acteur principal. Le pouvoir disciplinaire et l'homophobie s'ancrent et s'infiltrent dans le sujet par le biais des affects, des sentiments et des gestes de façon pour devenir la machine de destruction de son être intérieur. Le but ultime de ce

processus hétéronormatif de discipline et de régulation est l'autodestruction de la subjectivité queer.

\section{L'autodestruction du sujet queer}

Du refus de la caresse de Nathan jusqu'à la destruction de ce dernier, nous pouvons considérer que Louis se retrouve dans un état de soumission affective face à l'hétéronormativité. Cet état est pourtant mis à l'épreuve au moment où la mère de Louis découvre de la pornographie homosexuelle sur l'ordinateur de son fils. C'est alors que ses deux parents le confrontent. Louis demeure silencieux et vaincu, visiblement tiraillé entre l'amour qu'il ressent pour Nathan, et le regard percutant de ses parents qui ne veulent pas que leur garçon soit homosexuel (48:57-49:34). Détectant sa fragilité, le père de Louis assume le rôle du bourreau hétéronormatif, allant jusqu'à le frapper violemment lors d'une séance de boxe pour détruire symboliquement ses désirs (50:3852:37). Il l'enferme aussi dans sa chambre (01:16:05). De plus, il l'isole de sa mère et de son petit frère en lui interdisant tout contact affectif (58:48-01:00:46). Le père de Louis coupe donc son fils de la douceur, de la vulnérabilité et de la tendresse. Bien que le père ait conscience de l'extrémité de ses mesures, il s'évertue à discipliner et à punir son fils, insistant sur le fait que c'est à eux [le père et la mère] « de lui montrer qu'il se trompe » afin qu'il ne « gâche pas sa vie » (53:13-53:30). Nous pouvons alors déduire que le père, qui d'ailleurs est un psychiatre, a intériorisé l'idée que l'épanouissement passe uniquement par la performance d'une identité hétérosexuelle. Le père luimême est incapable de se libérer des chaînes de la discipline hétéronormative qui l'oblige en quelque sorte à rejeter son fils qui est gay. 
Nous observons l'impact de la mise en place et de la reproduction de ce mécanisme de contrôle affectif lorsque Louis, dans une dernière tentative pour « se guérir » et se libérer pour un temps de cette emprise normative, se retrouve seul à boxer agressivement un ballon de frappe et à se fixer dans le miroir, répétant à plusieurs reprises en voix-off : «Je suis pas homo, je suis pas homo, je suis pas homo...» avant de donner un coup de poing au miroir (55:52-56:23). Contrairement au début du film où il semble maîtriser la performance de l'hétéromasculinité pensons notamment au premier refus catégorique de la caresse de Nathan - Louis semble désormais incapable de contrôler son affectivité, voulant à tout prix intégrer les normes hétérosexuelles et anéantir sa subjectivité gay. L'acte de fixer son reflet dans le miroir lui permet aussi de voir son propre déni, de le ressentir, mais de ne jamais pouvoir se libérer de son emprise qui l'habite et le suit comme son ombre. Louis essaie de se convaincre qu'il est libre de ses choix, en les libérant par la parole, mais en fait il est enfermé comme le cadre du miroir qui rappelle symboliquement qu'il ne doit pas dépasser les normes sociétales. Ainsi, la peur maintient Louis dans un état de soumission à l'hétéronormativité en éclipsant sa volonté et en le conditionnant, tel un chien de Pavlov, à produire la performance hétérosexuelle qu'il a été conditionné à performer.

Étant incapable de contrôler son affectivité sous un registre hétéronormatif, Louis, à l'instar de plusieurs personnes queer, envisage de se suicider pour échapper à la prison de la société hétéronormative (1:02:40). Cette autodestruction s'inscrit dans la logique hétéronormative, binaire et patriarcale qui discipline l'individu à internaliser l'idée que s'il n'est pas un homme hétérosexuel, il doit se suicider ${ }^{8}$. Dans la tentative de suicide de Louis se conjuguent alors la discipline et l'emprise hétéronormatives : la force affective qu'il ressent atteint un tel point qu'il n'est plus capable de s'interdire le désir qu'il éprouve pour Nathan, voire de contrôler son affectivité queer, et par conséquent il croit qu'il lui reste seulement le suicide pour échapper à la prison de la discipline hétéronormative. L'autodestruction du sujet queer pourrait donc être interprétée comme une tentative pour s'émanciper de la matrice hétérosexuelle. De même, cette force autodestructrice est un appel à reconnaître la légitimité des affects queer qui habitent le corps et l'esprit de Louis.

Notons néanmoins que le fait que Louis ressente un réel besoin de mettre fin à sa vie plutôt que de demeurer prisonnier des normes hétérosexuelles nous permet de constater que l'affect queer est irréductiblement incontrôlé et incontrôlable. Autrement dit, pour reprendre l'idée de Jack Halberstam dans The Queer Art of Failure (2011), c'est précisément par son échec à performer 
correctement les normes hétérosexuelles que Louis échappe jusqu'à un certain point à la discipline hétéronormative dans la mesure où son affectivité queer ne peut plus être ni normalisée ni contrôlée, comme en témoigne sa décision de finalement céder à la tendresse de Nathan en choisissant de prendre sa main, plutôt que de se suicider (1:06:23). Sachant qu'il n'arrive pas à être hétérosexuel, Louis doit trouver d'autres moyens pour se légitimer dans un monde qui lui impose des attentes auxquelles son identité sexuelle ne pourra jamais correspondre. Cet échec crée alors les conditions de possibilité de la resubjectivation gay, sans pour autant éviter l'idée de l'autodestruction physique et identitaire dont plusieurs personnes queer sont souvent victimes.

\section{Conclusion}

Dans Baisers cachés, la discipline et la régulation hétéronormatives du sujet queer passent à travers le pouvoir de l'affect en assujettissant le sujet à des normes qu'il intériorise et performe, non seulement dans les espaces publics, où ses actions sont soumises au jugement des autres, mais également dans les espaces intimes, là où pourtant il pourrait se permettre d'agir conformément à ce qu'il ressent. Dans une société où les vies hétérosexuelles et binaires sont valorisées, il paraît évident pour Louis de survivre au regard des autres en se pliant aux normes hétéronormatives afin d'éviter le rejet, la marginalisation, la stigmatisation et l'exclusion. Dans le cas de Louis, s’identifier comme étant hétérosexuel est nécessaire pour avoir l'illusion de se sentir épanoui, alors que dans une optique hétéronormative assumer son identité gay est intimement lié à la peur et à l'échec. Ces affects, positifs et négatifs, correspondent à des sentiments et à des émotions que le sujet queer intériorise dans l'espace public hétéronormatif et qu'il actualise par le biais d'une performance sociale afin de profiter des privilèges patriarcaux, masculins et hétérosexuels. De ce fait, les sentiments de la peur et de l'épanouissement, en tant que mécanismes de contrôle individuel, sont le résultat d'interactions et de discours affectifs opérant ensemble dans les sphères sociales, culturelles et institutionnelles. Le contrôle social des affects a pour but de dresser l'individu pour qu'il devienne lui-même un agent actif au service du système hétéronormatif.

Bien que cet article n'ait pas eu pour but d'analyser la manière dont les adolescent.e.s du secondaire sont affecté.e.s par le film Baisers cachés et les types de réactions que les affects mis en jeu dans ce film peuvent déclencher chez ce public cible, il convient néanmoins de réfléchir à la possibilité qui s'ouvre - pensons notamment dans le contexte pédagogique de la salle de classe - lorsqu'on accepte, reconnaît et partage l'expérience affective de l'emprise comme partie 
intégrante du cheminement de la subjectivité queer. En quoi le fait de faire circuler, auprès d'adolescent.e.s du secondaire, l'expérience affective de la peur, a-t-il un pouvoir émancipateur pour les adolescent.e.s queer ? Cette question est notamment abordée dans Baisers cachés au moment où l'enseignante de mathématiques, Catherine, fait son « coming-out » devant ses élèves et raconte la peur qu'elle ressentait en tant que jeune fille lesbienne à l'école (41:29-44:03). À travers le partage d'une souffrance commune avec Nathan et d'autres élèves qui n'osent peut-être pas sortir du placard, Catherine met de l'avant la souffrance causée par l'homophobie afin de valider et de faire exister cette expérience affective dans l'espace public. C'est d'ailleurs précisément ce moment dans le film qui semble avoir déstabilisé la discipline de Louis en lui faisant prendre conscience de son état de soumission à l'hétéronormativité et des possibilités qui s'ouvrent lorsqu'on s'assume pleinement. Cette mise en abîme nous permet de constater la manière dont l'affectivité queer fait éclater la discipline hétéronormative en déplaçant dans l'espace public la soi-disant objectivité hétéronormative au profit d'une subjectivité dissidente qui s'articule autour de l'emprise, la haine de soi et l'homophobie internalisée. Pour ces raisons, si la soumission en tant qu'expérience affective est un mécanisme de contrôle social, elle peut être détournée par des actes et discours émancipatoires qui ont la capacité de libérer la parole queer et de légitimer des subjectivités et des formes de relations qui vont au-delà de celles que sanctifie la société hétéronormative.

\section{Bibliographie}

Agence France-Presse. «L'évolution des droits des homosexuels en France ». L'Express, 19 avril 2018. https://www.lexpress.fr/actualites/1/styles/l-evolution-des-droits-deshomosexuels-en-france_2001271.html. Consulté le 9 septembre.

Allo Docteurs. " "Baisers cachés" : des ados face à l'homophobie - Le Magazine de la santé ». YouTube, 17 mai 2017. https://www.youtube.com/watch?v=5E8bUyNG9jY. Consulté le 20 janvier 2019.

Baisers cachés. Réalisateur Didier Bivel. Breaking Glass Pictures, 2016.

Butler, Judith. Gender Trouble. New York : Routledge, 1990.

Cauhapé, Véronique. " "Baisers cachés", le calvaire de deux garçons qui s'aiment ». Le Monde, 17 mai 2017. https://www.lemonde.fr/televisions-radio/article/2017/05/17/tv-baiserscaches-le-calvaire-de-deux-garcons-qui-s-aiment_5129350_1655027.html. Consulté le 20 janvier 2019. 
Dubois, Arthur. « Bérenger Anceaux se livre sur le tournage de Baisers Cachés ». Le Figaro, 19 mai 2017. http://tvmag.lefigaro.fr/programme-tv/berenger-anceaux-se-livre-sur-letournage-de-baisers-caches_6ee84b02-3bcd-11e7-b5b5-21a5cdc791d1/. Consulté le 20 janvier 2019.

Duncan, Neil. «"It's important to be nice, but it's nicer to be important" : Girls, Popularity and Sexual Competition ». Sex Education 4.2 (2004). 137-152.

Foucault, Michel. Surveiller et punir: Naissance de la prison. Paris : Gallimard, 1975.

Green, Amy E., Myeshia Price-Feeney et Samuel H. Dorison. National Estimate of LGBTQ Youth Seriously Considering Suicide. New York : The Trevor Project, 2019.

Halberstam, Jack. The Queer Art of Failure. Durham / Londres : Duke UP 2011.

Haskell, Rebecca et Brian Burtch. Get that Freak : Homophobia and Transphobia in High Schools. Halifax / Winnipeg : Fernwood, 2010.

Hedrich, Pierre. «"Baisers cachés” : portrait de l'homophobie ordinaire ». TéléObs, 17 mai 2019. https://teleobs.nouvelobs.com/la-selection-teleobs/20170516.OBS9470/baiserscaches-portrait-de-1-homophobie-ordinaire.html. Consulté le 9 septembre 2019. Hullot-Guiot, Kim. «"Baisers cachés”, Quand une fiction télé parle d'homophobie au lycée ». Libération, 17 mai 2017. https://next.liberation.fr/culture/2017/05/17/baisers-cachesquand-une-fiction-tele-parle-d-homophobie-au-lycee_1570218. Consulté le 20 janvier 2019.

Kjaran, Jón Ingvar. Constructing Sexualities and Gendered Bodies in School Space : Nordic Insights on Queer and Transgender Students. New York, NY: Palgrave Macmillan, 2017.

Mac an Ghaill, Máirtín. «Schooling, Sexuality and Male Power. Towards an Emancipatory Curriculum ». Gender and Education 3.3 (1991). 291-309.

Massumi, Brian. Politics of Affect. Cambridge : Polity, 2015.

Pugniere, Jean-Michel. « Suicide des jeunes et homophobie en France : présentation d'une enquête et d'actions de prévention ». Service social 59.1 (2013). 17-34.

Richard, Gabrielle. Hétéro, l'école ? Plaidoyer pour une éducation antioppressive à la sexualité. Montréal : Remue-ménage, 2019. 
Rubin, Gayle. «Thinking Sex : Notes for a Radical Theory of the Politics of Sexuality ». Pleasure and Danger : Exploring Female Sexuality. Dir. Carole S. Vance. Routledge / Kegan Paul, 1984. 267-319. Sedgwick, Eve Kosofsky. Epistemology of the Closet. Berkeley : UCalifornia P, 1990. SOS homophobie. Rapport sur l'homophobie 2019, 2019. https://www.soshomophobie.org/sites/default/files/rapport_homophobie_2019_interactif.pdf

\footnotetext{
Notes

${ }_{1}$ «Quant aux agressions physiques rapportées à SOS homophobie, leur nombre est passé de 139 en 2017 à 231 en 2018, soit une augmentation de $66 \%$ \% (SOS homophobie 7). SOS homophobie est l'association nationale de lutte en France contre la lesbophobie, la gayphobie, la biphobie et la transphobie.

2 Précisons que malgré la décriminalisation de l'homosexualité en 1791 en France, la sanction pénale de l'homosexualité impliquant des personnes de plus de quinze ans (majorité sexuelle) n'est pas supprimée avant 1982. Or, il a fallu attendre 2004 pour que la loi française pénalise les propos homophobes (Agence France-Presse).

${ }^{3}$ Diverses études remontant jusqu'aux années 1990 ont montré la manière dont l'école en tant qu'institution hétéronormative constitue un mécanisme de surveillance de la sexualité et de la performance du genre des élèves. Voir, entre autres, les travaux de Máirtín Mac an Ghaill (1991), de Neil Duncan (2004), de Rebecca Haskell et Brian Burtch (2010), de Jón Ingvar Kjaran (2017) et de Gabrielle Richard (2019).

${ }^{4}$ L'impact de la droite catholique française se fait également ressentir dans la sphère éducative, se mobilisant contre toute tentative de rendre l'éducation à la sexualité plus inclusive. Comme le constate Gabrielle Richard, « [l'importance] et le nombre des rassemblements témoignent de la résistance encore bien ancrée à de tels changements, ainsi que de la capacité de mobilisation de la droite française et des associations catholiques » (55).

${ }^{5}$ Ce n'est qu'en 2016 que la loi française permet aux personnes homosexuelles de donner leur sang, et ce, à condition qu'elles s'abstiennent de rapports sexuels pendant un an (Agence France-Presse). L'homosexualité n'est plus considérée comme une maladie mentale, certes, mais cette loi conservatrice, qui ne s'applique pas aux personnes hétérosexuelles, discrimine en fonction de l'identité et de pratiques sexuelles prétendument risquées. Cela suggère qu'à de nombreux égards, l'homosexualité est encore perçue, bien que de manière insidieuse, comme une pathologie. 6 Massumi considère que les structures d'oppression constituent des régularités de capture, c'est-à-dire des mécanismes de pouvoir circulaires - pensons notamment à l'exemple de l'institution scolaire - dont la dissémination dépend d'une certaine complicité de ce qui lui est extérieur. Ainsi, Massumi place le pouvoir de l'affect dans un espace intersubjectif, interrelationnel, interactif et fortement physique, concret et corporel : «Structures of power are powered by these different-order movements that they capture. They are parasitic. Vampiric. They emerge, self-structuring, surfing the crest of outside energies, and can only perpetuate themselves by diving in and catching the wave again. They have no motive force of their own » (102). Cela laisse entendre que l'hétéronormativité en tant que structure de pouvoir puise son énergie dans les relations qu'elle entretient avec des sujets consentants et soumis, comme l'illustre le cas de Louis.

${ }^{7}$ Le panoptique est une structure architecturale qui permet à un gardien d'observer, depuis une tour centrale, tous les prisonniers enfermés dans des cellules individuelles. Le but de l'appareil est de pouvoir observer le plus grand nombre de prisonniers à leur insu. D'un point de vue pratique, vu que les prisonniers se disciplinent simplement parce qu'ils croient être surveillés, cela élimine la nécessité de faire appel à plus de gardiens. Michel Foucault reprend le concept du panoptique pour en faire un modèle par excellence des institutions disciplinaires qui fonctionnent à la fois comme des dispositifs de surveillance et d'auto-surveillance. Il écrit : «De là, l'effet majeur du Panoptique : induire chez le détenu un état conscient et permanent de visibilité qui assure le fonctionnement automatique du pouvoir. Faire que la surveillance soit permanente dans ses effets, même si elle est discontinue dans son action ; que la perfection du pouvoir tende à rendre inutile l'actualité de son exercice ; que cet appareil architectural soit une machine à créer et à soutenir un rapport de pouvoir indépendant de celui qui l'exerce ; bref que les détenus soient pris dans une situation de pouvoir dont ils sont eux-mêmes les porteurs. Pour cela, c'est à la fois trop et trop peu que le prisonnier soit sans cesse observé par un surveillant : trop peu, car l'essentiel c'est qu'il se sache surveillé ; trop, parce qu'il n'a pas besoin de l'être effectivement $\gg(234-235)$.

${ }^{8}$ Une enquête menée en France auprès de 901 jeunes garçons et filles sous forme de questionnaire constate le lien entre le suicide et l'homophobie en milieu scolaire. Publiée en 2013, l'étude a non seulement montré que les idéations
} 
et les tentatives de suicide étaient plus présentes chez les participant.es LGBQ, mais elle a aussi révélé que le risque de suicide est tributaire de l'intimidation verbale homophobe et de l'intimidation basée sur la non-conformité de genre, surtout chez les jeunes hommes (Pugniere 25-26). Ce phénomène de sursuicidalité est d'ailleurs confirmé par un rapport publié en 2019 par le Trevor Project, qui estime qu'au moins 1,2 million de jeunes LGBTQ âgé.e.s de treize à dix-huit ans aux États-Unis pensent sérieusement au suicide chaque année (Green et al.). Ce ne sont que deux exemples qui permettent de mieux comprendre l'ampleur des pensées et des comportements suicidaires chez les jeunes LGBTQ. 